\title{
OPERATORS WITH COMPLEX GAUSSIAN KERNELS: BOUNDEDNESS PROPERTIES
}

\author{
E. R. NEGRIN
}

(Communicated by Palle E. T. Jorgensen)

\begin{abstract}
Boundedness properties are stated for some operators from $L^{p}(\mathbb{R})$ into $L^{q}(\mathbb{R}), 1 \leq p, q \leq \infty$, with complex Gaussian kernels. Their contraction properties are also analysed.
\end{abstract}

\section{INTRODUCTION}

In this paper we will study boundedness properties for the general complex Gaussian operator in dimension one,

$$
\left(\mathscr{F}_{\beta, \varepsilon, \delta, \xi, \gamma} f\right)(x)=\int_{-\infty}^{+\infty} \exp \left\{-\beta x^{2}-\varepsilon y^{2}+2 \delta x y+\xi x+\gamma y\right\} \cdot f(y) d y
$$

$\beta, \varepsilon, \delta, \xi, \gamma \in \mathbb{C}, x \in \mathbb{R}$, from the space of complex-valued functions $L^{p}(\mathbb{R})$ into $L^{q}(\mathbb{R}), 1 \leq p, q \leq \infty$, relative to the Lebesgue measure.

This subject was originally of interest in the context of Quantum Field Theory (see [1]). The complex Gaussian operator (1.1) has an intrinsic interest due to the basic role of the extended oscillator semigroup introduced by Howe [4] (see also Folland [3, Chapter 5]).

In his important paper [5], Lieb extends the operator (1.1) to $n$ dimensions and develops an extensive study of $(1.1)$ in the context of the spaces $L^{p}\left(\mathbb{R}^{n}\right)$, $1<p<\infty$. Moreover, he generalizes the results given by Epperson in $\S 2$ of [2] for (1.1). Lieb stated that for the nondegenerate case, that is, $(\operatorname{Re} \delta)^{2}<(\operatorname{Re} \beta)$. $(\operatorname{Re} \varepsilon), \mathscr{F}_{\beta, \varepsilon, \delta, \xi, \gamma}$ has exactly one maximizer which is a centered Gaussian function $e^{s y^{2}}, s \in \mathbb{C}$. For the degenerate case, that is, $(\operatorname{Re} \delta)^{2}=(\operatorname{Re} \beta) \cdot(\operatorname{Re} \varepsilon)$, the question of the existence of a maximizer is a subtle one. This problem requires a complicated algebraic study, and precise conditions are not given there.

The purpose of our paper is to calculate the exact region of boundedness of (1.1) for each $1 \leq p, q \leq \infty$, in both degenerate and nondegenerate cases.

Received by the editors November 9, 1992 and, in revised form, July 15, 1993; part of the results of this paper have been presented to the First Congress of Mathematics, Paris, July 6-10, 1992, European Mathematical Society.

1991 Mathematics Subject Classification. Primary 47B38.

Key words and phrases. Lebesgue measure, bounded operator, contraction, Gaussian kernels. 
The proof of this result follows a technique initiated by Weissler in Theorem 1 of [6]. Weissler sated the exact region of boundedness for the Hermite semigroup.

We conclude this paper by giving sufficient conditions in both degenerate and nondegenerate cases for the operator $\mathscr{F}_{\beta, \varepsilon, \delta, \xi, \gamma}, \beta, \varepsilon, \delta, \xi, \gamma \in \mathbb{R}$, to be a contraction over $L^{p}(\mathbb{R}), 1<p<\infty$, and a contraction as an operator from $L^{2}(\mathbb{R})$ into $L^{p}(\mathbb{R}), 0<p<\infty$. These results follow those given in Chapter 8 of [1].

Throughout this paper we will take square roots with positive real part.

\section{BOUNDEDNESS PROPERTIES}

For $1 \leq p \leq \infty$, let $\left(I_{p} f\right)(x)=(2 \pi)^{-1 / 2 p} \cdot \exp \left(-x^{2} / 2 p\right) \cdot f(x)$. For $z \in \mathbb{C}$, let $\left(Q_{z} f\right)(x)=e^{z x} \cdot f(x)$. For $\gamma^{*}>0$, let $\left(T_{\gamma^{*}} f\right)(x)=f\left(\gamma^{*} x\right)$. Also, for $\alpha^{*} \in \mathbb{C}$, let $\left(M_{\alpha^{*}} f\right)(x)=\exp \left(-\alpha^{*} x^{2} / 2\right) \cdot f(x)$. The Gauss-Weierstrass semigroup on $\mathbb{R}$ is given by

$$
\left(e^{z \Delta} f\right)(x)=(4 \pi z)^{-1 / 2} \cdot \int_{-\infty}^{+\infty} \exp \left[-(x-y)^{2} / 4 z\right] \cdot f(y) d y, \quad x \in \mathbb{R},
$$

where $\operatorname{Re} z \geq 0$ (and $z \neq 0$ ). Finally, we denote by $\left\|\mathscr{F}_{\beta, \varepsilon, \delta, \varepsilon, \gamma}\right\|_{p, q}$ the norm of $\mathscr{F}_{\beta, \ell, \delta, \xi, \gamma}$ as a map from $L^{p}(\mathbb{R})$ into $L^{q}(\mathbb{R}), 1 \leq p, q \leq \infty$.

It is easy to check that for any $\gamma^{*}>0$ and $\operatorname{Re} \delta \geq 0$,

$$
\mathscr{F}_{\beta, \varepsilon, \delta, \xi, \gamma}=\left(\pi \gamma^{*} / \delta\right)^{1 / 2}(2 \pi)^{(1 / 2 p)-(1 / 2 q)} Q_{\xi} I_{q}^{-1} M_{\beta^{*}} T_{\gamma^{*}} e^{\left(\gamma^{*} / 4 \delta\right) \Delta} M_{\alpha^{*}} I_{p} Q_{\gamma},
$$

where

$$
\alpha^{*}=2 \varepsilon-\frac{1}{p}-\frac{2 \delta}{\gamma^{*}}, \quad \beta^{*}=2 \beta+\frac{1}{q}-2 \gamma^{*} \delta .
$$

We will denote by $q^{\prime}$ the exponent conjugate to $q$.

Theorem 2.1. The following hold:

(i) If $1 \leq p \leq q \leq \infty, \operatorname{Re} \delta>0$, and $\operatorname{Re} \varepsilon>0$, then $\mathscr{F}_{\beta, \varepsilon, \delta, \xi, \gamma}$ is bounded from $L^{p}(\mathbb{R})$ to $L^{q}(\mathbb{R})$ if and only if

$$
(\operatorname{Re} \varepsilon) \cdot(\operatorname{Re} \beta) \geq(\operatorname{Re} \delta)^{2} .
$$

(ii) If $1 \leq q<p \leq \infty, \operatorname{Re} \delta>0$, and $\operatorname{Re} \varepsilon>0$, then $\mathscr{F}_{\beta, \varepsilon, \delta, \xi, \gamma}$ is bounded from $L^{p}(\mathbb{R})$ to $L^{q}(\mathbb{R})$ if and only if

$$
(\operatorname{Re} \varepsilon) \cdot(\operatorname{Re} \beta)>(\operatorname{Re} \delta)^{2} .
$$

(iii) If $1 \leq p, q \leq \infty, \operatorname{Re} \delta=0, \frac{1}{4}-\frac{1}{2 p} \leq \operatorname{Re} \varepsilon \leq \frac{1}{2}-\frac{1}{2 p}$, and $\operatorname{Re} \beta \geq$ $\operatorname{Re} \varepsilon+\frac{1}{2 p}-\frac{1}{2 q^{\prime}} \geq 0$, then $\mathscr{T}_{\beta, \varepsilon, \delta, \xi, \gamma}$ is bounded from $L^{p}(\mathbb{R})$ to $L^{q}(\mathbb{R})$ if and only if $\operatorname{Re} \varepsilon \geq 0$.

(iv) If $1 \leq p, q \leq \infty, \operatorname{Re} \delta=0, \frac{1}{2 p}-\frac{1}{2 q^{\prime}} \leq \operatorname{Re} \beta \leq \operatorname{Re} \varepsilon+\frac{1}{2 p}-\frac{1}{2 q^{\prime}}$, and $\frac{1}{4}-\frac{1}{2 q^{\prime}} \leq \operatorname{Re} \beta \leq \frac{1}{2}-\frac{1}{2 q^{\prime}}$, then $\mathscr{F}_{\beta, \varepsilon, \delta, \xi, \gamma}$ is bounded from $L^{p}(\mathbb{R})$ to $L^{q}(\mathbb{R})$ if and only if $\operatorname{Re} \varepsilon \geq 0$ and $\operatorname{Re} \beta \geq 0$.

Proof. Suppose first that $\operatorname{Re} \delta=0, \frac{1}{4}-\frac{1}{2 p} \leq \operatorname{Re} \varepsilon \leq \frac{1}{2}-\frac{1}{2 p}, \operatorname{Re} \beta \geq \operatorname{Re} \varepsilon+\frac{1}{2 p}-$ $\frac{1}{2 q^{\prime}} \geq 0$, and $1 \leq p, q \leq \infty$. Now, if $\operatorname{Re} \varepsilon \geq 0, \operatorname{Re} \beta \geq 0$, and $m$ is a real number such that $\operatorname{Re} 2 \varepsilon+\frac{1}{p}=\frac{1}{m}$, we have $1 \leq m \leq 2$, and the Hausdorff-Young inequality yields that $e^{\left(\gamma^{*} / 4 \delta\right) \Delta}$ is bounded from $L^{m}(\mathbb{R})$ into $L^{m^{\prime}}(\mathbb{R})\left(m^{\prime}\right.$ is the 
exponent conjugate to $m$ ). But from the hypothesis $\mathscr{F}_{\beta-(1 / 2 q), \varepsilon+(1 / 2 p), \delta, \xi, \gamma}$ is bounded from $L^{m}\left(\mathbb{R}, e^{-x^{2} / 2} d x\right)$ into $L^{m^{\prime}}\left(\mathbb{R}, e^{-x^{2} / 2} d x\right)$. Since $p \geq m$ and $q \leq m^{\prime}$, the operator $\mathscr{F}_{\beta-(1 / 2 q), \varepsilon+(1 / 2 p), \delta, \xi, \gamma}$ is bounded from $L^{p}\left(\mathbb{R}, e^{-x^{2} / 2} d x\right)$ into $L^{q}\left(\mathbb{R}, e^{-x^{2} / 2} d x\right), 1 \leq p, q \leq \infty$, and therefore $\mathscr{F}_{\beta, \varepsilon, \delta, \xi, \gamma}$ is bounded from $L^{p}(\mathbb{R})$ into $L^{q}(\mathbb{R})$.

For the case $\operatorname{Re} \delta=0, \frac{1}{2 p}-\frac{1}{2 q^{\prime}} \leq \operatorname{Re} \beta \leq \operatorname{Re} \varepsilon+\frac{1}{2 p}-\frac{1}{2 q^{\prime}}, \frac{1}{4}-\frac{1}{2 q^{\prime}} \leq \operatorname{Re} \beta \leq$ $\frac{1}{2}-\frac{1}{2 q^{\prime}}$, and $1 \leq p, q \leq \infty$, the proof is similar.

Now assume $\operatorname{Re} \delta>0$, so that $\operatorname{Re}\left(\gamma^{*} / 4 \delta\right)>0$ and therefore, for $1 \leq$ $p \leq q \leq \infty, e^{\left(\gamma^{*} / 4 \delta\right) \Delta}$ is bounded from $L^{p}(\mathbb{R})$ into $L^{q}(\mathbb{R})$. Since $\operatorname{Re} \varepsilon \geq 0$, $\operatorname{Re} \beta \geq 0$, and $(\operatorname{Re} \varepsilon) \cdot(\operatorname{Re} \beta) \geq(\operatorname{Re} \delta)^{2}, \gamma^{*}$ can be chosen so that $\operatorname{Re} \alpha^{*} \geq 0$ and $\operatorname{Re} \beta^{*} \geq 0$. It follows from $(2.1)$ that $\mathscr{F}_{\beta-(1 / 2 q), \varepsilon+(1 / 2 p), \delta, \xi, \gamma}$ is bounded from $L^{p}\left(\mathbb{R}, e^{-x^{2} / 2} d x\right)$ into $L^{q}\left(\mathbb{R}, e^{-x^{2} / 2} d x\right), 1 \leq p, q \leq \infty$, and hence $\mathscr{F}_{\beta, \varepsilon, \delta, \xi, \gamma}$ is bounded from $L^{p}(\mathbb{R})$ into $L^{q}(\mathbb{R})$.

For the case $1 \leq q<p \leq \infty$ and from the conditions $\operatorname{Re} \varepsilon \geq 0, \operatorname{Re} \beta \geq 0$, and $(\operatorname{Re} \varepsilon) \cdot(\operatorname{Re} \beta)>(\operatorname{Re} \delta)^{2}, \gamma^{*}$ can be chosen so that $\operatorname{Re} \alpha^{*}>0$ and $\operatorname{Re} \beta^{*}>0$. Observing that $M_{\beta^{*}}$ is a bounded map from $L^{p}(\mathbb{R})$ into $L^{q}(\mathbb{R})$ for $\operatorname{Re} \beta^{*}>0$ and since $e^{\left(\gamma^{*} / 4 \delta\right) \Delta}$ is bounded over $L^{p}(\mathbb{R})$, equality (2.1) implies that $\mathscr{F}_{\beta-(1 / 2 q), \varepsilon+(1 / 2 p), \delta, \xi, \gamma}$ is bounded from $L^{p}\left(\mathbb{R}, e^{-x^{2} / 2} d x\right)$ into $L^{q}\left(\mathbb{R}, e^{-x^{2} / 2} d x\right), 1 \leq q<p \leq \infty$. Therefore, $\mathscr{F}_{\beta, \varepsilon, \delta, \xi, \gamma}$ is bounded from $L^{p}(\mathbb{R})$ into $L^{q}(\mathbb{R})$.

In order to prove the converse, suppose $\left\|\mathscr{F}_{\beta, \varepsilon, \delta, \xi, \gamma}\right\|_{p, q}<\infty$. We will prove that $\operatorname{Re} \varepsilon \geq 0, \operatorname{Re} \beta \geq 0$, and $(\operatorname{Re} \varepsilon) \cdot(\operatorname{Re} \beta) \geq(\operatorname{Re} \delta)^{2}$ and that $(\operatorname{Re} \varepsilon) \cdot$ $(\operatorname{Re} \beta)>(\operatorname{Re} \delta)^{2}$ holds if $q<p$. To this end, we need to calculate the action of $\mathscr{F}_{\beta, \varepsilon, \delta, \xi, \gamma}$ on an arbitrary Gaussian function $g_{s}(y)=e^{s y^{2}}, s \in \mathbb{C}, y \in \mathbb{R}$. Then $\mathscr{F}_{\beta, \varepsilon, \delta, \xi, \gamma}$ can be computed for $\operatorname{Re} s<\operatorname{Re} \varepsilon$ to obtain

$$
\begin{aligned}
& \left(\mathscr{F}_{\beta, \varepsilon, \delta, \xi, \gamma} g_{s}\right)(x) \\
& \quad=\left(\frac{\pi}{\varepsilon-s}\right)^{1 / 2} \cdot \exp \left(\frac{\delta^{2}-\beta \varepsilon+\beta s}{\varepsilon-s} x^{2}+\frac{\delta \gamma+\xi \varepsilon-\xi s}{\varepsilon-s} x+\frac{\gamma^{2}}{4(\varepsilon-s)}\right),
\end{aligned}
$$

with $x \in \mathbb{R}$.

We impose that $g_{s} \in L^{p}(\mathbb{R})$ so that $\operatorname{Re} s<0$. Now, we want (2.2) to be in $L^{q}(\mathbb{R})$. With this purpose, let us consider the transformation $L(s)$, given by

$$
L(s)=\frac{\delta^{2}-\beta \varepsilon+\beta s}{\varepsilon-s} .
$$

If $\mathscr{F}_{\beta, \varepsilon, \delta, \xi, \gamma} \in L^{q}(\mathbb{R})$, then $\operatorname{Re} L(s)<0$. Note that $L$ maps $\varepsilon$ to $\infty$. Therefore, given the line $\operatorname{Re} s=0$, there exists a circle $C$ passing through $\varepsilon$ such that $L$ applies $C$ into the line $\operatorname{Re} s=0$. We claim that $\operatorname{Re} \varepsilon \geq 0$. In fact, assume that $\operatorname{Re} \varepsilon<0$. Then $\operatorname{Re} s<\operatorname{Re} \varepsilon<0$. Let $s_{0}$ be a point of the circle $C$ satisfying $\operatorname{Re} s_{0}<0$ and $\operatorname{Re} L\left(s_{0}\right)=0$. Assume that $s \rightarrow s_{0}$ with the restrictions $\operatorname{Re} s \leq-\varepsilon^{*} \quad\left(\varepsilon^{*}>0\right)$ and $\operatorname{Re} L(s)<0$. Then $g_{s}$ remains bounded in $L^{p}(\mathbb{R})$ as $s \rightarrow s_{0}$, while $\mathscr{F}_{\beta, \varepsilon, \delta, \xi, \gamma}$ blows up in $L^{q}(\mathbb{R})$. This is a contradiction because $\mathscr{F}_{\beta, \varepsilon, \delta, \xi, \gamma}$ is bounded, and we conclude that $\operatorname{Re} \varepsilon \geq 0$.

In order to verify that $\operatorname{Re} \beta \geq 0$, let

$$
L_{1}(s)=L(s)+\beta=\frac{\delta^{2}}{\varepsilon-s} .
$$


Taking $\operatorname{Re} s=-\varepsilon^{*}\left(\varepsilon^{*}>0\right)$ and noting that $\operatorname{Re} s<0$ implies $\operatorname{Re} L(s)<0$, we have

$$
\operatorname{Re} L_{1}(s)=\operatorname{Re} L(s)+\operatorname{Re} \beta \leq \operatorname{Re} \beta .
$$

Now, letting $s$ tend to infinity along the line $\operatorname{Re} s=-\varepsilon^{*}$, we see that $0 \leq$ $\lim _{s \rightarrow s_{0}} \operatorname{Re} L_{1}(s) \leq \operatorname{Re} \beta$, whence $\operatorname{Re} \beta \geq 0$. Thus $\operatorname{Re} \varepsilon \geq 0$ and $\operatorname{Re} \beta \geq 0$.

Next suppose that $\operatorname{Re} \varepsilon>0$. In this case $L_{1}$ carries the line $\operatorname{Re} s=0$ into a circle $C_{1}$ passing through 0 . By $(2.4)$, to prove that $(\operatorname{Re} \varepsilon) \cdot(\operatorname{Re} \beta) \geq(\operatorname{Re} \delta)^{2}$, it suffices to show that some point on that circle has real part

$$
[\operatorname{Re} \delta]^{2} \cdot[\operatorname{Re} \varepsilon]^{-1}
$$

Denote such a point by $s_{1}$. From (2.4) we obtain

$$
\operatorname{Re} L_{1}\left(s_{1}\right) \leq \operatorname{Re} \beta,
$$

so that $(\operatorname{Re} \varepsilon) \cdot(\operatorname{Re} \beta) \geq(\operatorname{Re} \delta)^{2}$. The center of the circle $C_{1}$ is $\frac{1}{2} L_{1}\left(s_{2}\right)$, where $s_{2}$ minimizes $|\varepsilon-s|$ subject to the condition $\operatorname{Re} s=0$. It is easy to check that $\frac{1}{2} L_{1}\left(s_{2}\right)+\left|\frac{1}{2} L_{1}\left(s_{2}\right)\right|$ has the desired real part.

Finally, if $q<p$, we must show that the equality cannot hold in $(\operatorname{Re} \varepsilon) \cdot$ $(\operatorname{Re} \beta)>(\operatorname{Re} \delta)^{2}$. Indeed, if it did, then $\gamma^{*}$ could be chosen so that $\operatorname{Re} \alpha^{*}=$ $\operatorname{Re} \beta^{*}=0$ in (2.1). Then (2.1) would imply that $e^{\left(\gamma^{*} / 4 \delta\right) \Delta}$, with $\operatorname{Re}\left(\gamma^{*} / 4 \delta\right)>0$, is bounded from $L^{p}(\mathbb{R})$ into $L^{q}(\mathbb{R})$, which is false.

\section{CONTRACTION PROPERTIES}

The purpose of this section is to give sufficient conditions in order that the operator $\mathscr{F}_{\beta, \varepsilon, \delta, \xi, \gamma}$ (for degenerate and nondegenerate cases), $\beta, \varepsilon, \delta, \xi, \gamma \in$ $\mathbb{R}$, be a contraction over $L^{p}(\mathbb{R}), 1<p<\infty$, and a contraction as an operator from $L^{2}(\mathbb{R})$ into $L^{p}(\mathbb{R}), 0<p<\infty$.

The next results are motivated by Chapter 8 of [1].

Theorem 3.1. Let $1<p<\infty$, and assume $\varepsilon>0$ and $\delta^{2}<p^{\prime} \beta \varepsilon$ (here, $p^{\prime}$ denotes the exponent conjugate to $p$ ). For all $f \in L^{p}(\mathbb{R})$, we have

$$
\begin{array}{r}
\left\|\mathscr{F}_{\beta, \varepsilon, \delta, \xi, \gamma} f\right\|_{p}^{p} \leq H \cdot \int_{-\infty}^{+\infty} \exp \left\{\left[\delta^{2} /\left(\beta p-\left(p / p^{\prime}\right) \cdot\left(\delta^{2} / \varepsilon\right)\right)-\varepsilon\right] y^{2}\right. \\
+\left[\gamma+\left(\delta \cdot\left(\xi p+\left(p / p^{\prime}\right) \cdot(\gamma \delta / \varepsilon)\right)\right) /\left(\beta p-\left(p / p^{\prime}\right)\right.\right. \\
\left.\left.\left.\cdot\left(\delta^{2} / \varepsilon\right)\right)\right] y\right\} \cdot|f(y)|^{p} d y,
\end{array}
$$

where

$$
\begin{aligned}
H= & (\pi / \varepsilon)^{p / 2 p^{\prime}} \cdot\left(\pi /\left(\beta p-\left(p / p^{\prime}\right) \cdot\left(\delta^{2} / \varepsilon\right)\right)\right)^{1 / 2} \\
& \cdot \exp \left\{\left(p \gamma^{2} / 4 \varepsilon p^{\prime}\right)+\left(\left(\xi p+\left(p / p^{\prime}\right) \cdot(\gamma \delta / \varepsilon)\right)^{2}\right) /\left(4 \cdot\left(\beta p-\left(p / p^{\prime}\right) \cdot\left(\delta^{2} / \varepsilon\right)\right)\right)\right\} .
\end{aligned}
$$

Proof. By writing

$$
\begin{array}{r}
\left(\mathscr{F}_{\beta, \varepsilon, \delta, \xi, \gamma} f\right)(x)=\int_{-\infty}^{+\infty}\left\{\left(\exp \left[-\beta x^{2}-\varepsilon y^{2}+2 \delta x y+\xi x+\gamma y\right]\right)^{1 / p} \cdot f(y)\right\} \\
\cdot\left\{\left(\exp \left[-\beta x^{2}-\varepsilon y^{2}+2 \delta x y+\xi x+\gamma y\right]\right)^{1 / p^{\prime}}\right\} d y,
\end{array}
$$


$x \in \mathbb{R}$, and applying Hölder's inequality, it follows that

$$
\begin{aligned}
\left|\left(\mathscr{F}_{\beta, \varepsilon, \delta, \xi, \gamma} f\right)(x)\right| \leq & \left(\int_{-\infty}^{+\infty} \exp \left[-\beta x^{2}-\varepsilon y^{2}+2 \delta x y+\xi x+\gamma y\right] \cdot|f(y)|^{p} d y\right)^{1 / p} \\
& \cdot\left(\int_{-\infty}^{+\infty} \exp \left[-\beta x^{2}-\varepsilon y^{2}+2 \delta x y+\xi x+\gamma y\right] d y\right)^{1 / p^{\prime}} .
\end{aligned}
$$

Since for $\varepsilon>0$,

$$
\begin{aligned}
\int_{-\infty}^{+\infty} \exp \left[-\beta x^{2}-\varepsilon y^{2}+2 \delta x y+\xi y+\gamma x\right] d y \\
=(\pi / \varepsilon)^{1 / 2} \cdot \exp \left[\left(\left(\delta^{2} / \varepsilon\right)-\beta\right) x^{2}+(\xi+(\delta \gamma / \varepsilon)) x+\left(\gamma^{2} / 4 \varepsilon\right)\right],
\end{aligned}
$$

we arrive at the estimate

$$
\begin{aligned}
\left|\left(\mathscr{F}_{\beta, \varepsilon, \delta, \xi, \gamma} f\right)(x)\right|^{p} \\
\leq(\pi / \varepsilon)^{p / 2 p^{\prime}} \cdot \exp \left[p \gamma^{2} / 4 \varepsilon p^{\prime}\right] \\
\quad \cdot \int_{-\infty}^{+\infty} \exp \left[-\left(\beta p-\left(p / p^{\prime}\right) \cdot\left(\delta^{2} / \varepsilon\right)\right) x^{2}-\varepsilon y^{2}+2 \delta x y\right. \\
\left.\quad+\left(\xi p+\left(p / p^{\prime}\right) \cdot(\gamma \delta / \varepsilon)\right) x+\gamma y\right] \cdot|f(y)|^{p} d y .
\end{aligned}
$$

After integration with respect to $x$, the theorem follows.

Corollary 3.1. Under the same hypothesis and notation of Theorem 3.1, we set

$$
A=\delta^{2} /\left(\beta p-\left(p / p^{\prime}\right) \cdot\left(\delta^{2} / \varepsilon\right)\right)-\varepsilon
$$

and

$$
B=\gamma+\left(\delta \cdot\left(\xi p+\left(p / p^{\prime}\right) \cdot(\gamma \delta / \varepsilon)\right)\right) /\left(\beta p-\left(p / p^{\prime}\right) \cdot\left(\delta^{2} / \varepsilon\right)\right) .
$$

Then,

(a) If $A=0$ (or equivalently, $\delta^{2}=\beta \cdot \varepsilon$ ) and $B=0$, one has

$$
\left\|\mathscr{F}_{\beta, \varepsilon, \delta, \xi, \gamma} f\right\|_{p} \leq H^{1 / p} \cdot\|f\|_{p} \quad \text { for all } f \in L^{p}(\mathbb{R}) .
$$

Note that if $H \leq 1$, we obtain

$$
\left\|\mathscr{F}_{\beta, \varepsilon, \delta, \xi, \gamma} f\right\|_{p} \leq\|f\|_{p} \quad \text { for all } f \in L^{p}(\mathbb{R}) .
$$

(b) If $A<0$ (or equivalently, $\delta^{2}<\beta \cdot \varepsilon$ ), then

$$
\left\|\mathscr{F}_{\beta, \varepsilon, \delta, \xi, \gamma} f\right\|_{p} \leq\left(H \cdot \exp \left(B^{2} / 4 A\right)\right)^{1 / p} \cdot\|f\|_{p} \quad \text { for all } f \in L^{p}(\mathbb{R}) .
$$

Note that if $H \cdot \exp \left(B^{2} / 4 A\right) \leq 1$, we obtain

$$
\left\|\mathscr{F}_{\beta, \varepsilon, \delta, \xi, \gamma} f\right\|_{p} \leq\|f\|_{p} \text { for all } f \in L^{p}(\mathbb{R}) \text {. }
$$

Remark. For $1<p<\infty, \varepsilon>0, \delta \neq 0$, and $\delta^{2}=\beta \varepsilon$, the question of contractivity remains open if $B \neq 0$ or $H>1$. This question also remains open for $1<p<\infty, \varepsilon>0$, and $\delta^{2}<\beta \varepsilon$, if $H \cdot \exp \left(B^{2} / 4 A\right)>1$.

Theorem 3.2. Let $0<p<\infty$, and assume $\varepsilon>0$ and $\delta^{2}<\beta \varepsilon$. Then, for all $f \in L^{2}(\mathbb{R})$,

$$
\left\|\mathscr{F}_{\beta, \varepsilon, \delta, \xi, \gamma} f\right\|_{p} \leq H^{*} \cdot\|f\|_{2},
$$


where

$$
\begin{aligned}
H^{*}= & (2 \pi)^{1 / 4} \cdot(4 \varepsilon)^{-1 / 4} \cdot(\pi / p)^{1 / 2 p} \cdot\left(\beta-\left(\delta^{2} / \varepsilon\right)\right)^{-1 / 2 p} \\
& \cdot \exp \left\{\left(\gamma^{2} / 4 \varepsilon\right)+\left(p \cdot(\xi+(\delta \gamma / \varepsilon))^{2}\right) /\left(4 \cdot\left(\beta-\left(\delta^{2} / \varepsilon\right)\right)\right)\right\}
\end{aligned}
$$

Proof. By Schwarz's inequality and the evaluation of a Gaussian integral, we obtain for $f \in L^{2}(\mathbb{R})$,

$$
\begin{aligned}
\left|\left(\mathscr{F}_{\beta, \varepsilon, \delta, \xi, \gamma} f\right)(x)\right| \leq & (2 \pi)^{1 / 4} \cdot(4 \varepsilon)^{-1 / 4} \\
& \cdot \exp \left[\left(\left(\delta^{2} / \varepsilon\right)-\beta\right) x^{2}+(\xi+(\delta \gamma / \varepsilon)) x+\left(\gamma^{2} / 4 \varepsilon\right)\right] \cdot\|f\|_{2} .
\end{aligned}
$$

Again, by evaluating a Gaussian integral (3.2) follows.

Corollary 3.2. Under the same conditions and notation of Theorem 3.2 , if $H^{*} \leq$ 1 , then

$$
\begin{gathered}
\left\|\mathscr{F}_{\beta, \varepsilon, \delta, \xi, \gamma} f\right\|_{p} \leq\|f\|_{2} \text { for all } f \in L^{2}(\mathbb{R}) . \\
\text { REFERENCES }
\end{gathered}
$$

1. J. C. Baez, I. E. Segal, and Z. Zhou, Introduction to algebraic and constructive quantum field theory, Princeton Ser. Phys., Princeton Univ. Press, Princeton, NJ, 1992.

2. J. B. Epperson, The hypercontractive approach to exactly bounding an operator with complex Gaussian kernel, J. Funct. Anal. 87 (1989), 1-30.

3. G. B. Folland, Harmonic analysis in phase space, Ann. of Math. (2) 122 (1989).

4. R. E. Howe, The oscillator semigroup, The Mathematical Heritage of Hermann Weyl (Durham, NC, 1987), Proc. Sympos. Pure Math., vol. 48, Amer. Math. Soc., Providence, RI, 1988, pp. 61-132.

5. E. H. Lieb, Gaussian kernels have only Gaussian maximizers, Invent. Math. 102 (1990), 179-208.

6. F. B. Weissler, Two-point inequalities, the Hermite semigroup, and the Gauss-Weierstrass semigroup, J. Funct. Anal. 32 (1979), 102-121.

Deparatmento de Analisis Matematico, Universidad de la laguna, 38271, Canary IsLANDS, SPAIN

E-mail address: enegrin@ull.es 